\title{
Robotic Needle Felting
}

\section{TSZ NG}

University of Michigan

\author{
ASA PELLER \\ University of Michigan
}

Robotic Needle Felting explores the development of an additive manufacturing technique for nonwoven textiles. Nonwoven textiles, such as felt, can be natural materials (wool), synthetic polymers (polyester), or blends of the two. These textiles have numerous performative aspects for architectural applications including excellent acoustic absorption, thermal insulation, and tactile characteristics. The nonwoven textiles can be manipulated by a process called needle felting - whereby barbed needles, when punching through layers of material, entangle the fibers together making it a uniform material. This process binds the material together seamlessly without the addition of sewn thread or toxic adhesives, making this technique a more environmentally friendly process.

Needle felting can range in scale from handcraft techniques with a single needle to large scale web processing. Integration into a robotic process not only enables precision and speed in manufacturing but also extends needle felting as a threedimensional process, especially for surfaces with complex geometries and allowing for local differentiation of stiffness and other properties across a homogenous solid. Through a customized digital workflow, formal and material properties can be varied at local level within a component. By developing a fully integrated design to production methodology for influencing these properties, this research opens a wide range of potentials for nonwoven textiles in architectural applications.

The project involves three areas of development; the tooling for robotic felting, the digital workflow that enables the formal and material properties to be specified computationally and embedded into the machine code, and prototypes of architectural elements such as acoustic panels and furniture demonstrating different techniques and processes. Additive manufacturing (AM), commonly known as 3D printing, has revolutionized the design to production workflow in a wide range of disciplines. While AM processes have been developed for a wide range of materials, from ceramics to plastics to metals, there have been very few investigations into their applications for textiles. Given the unique capacity of felt to be seamlessly "added" into a cohesive solid, it presents a unique opportunity to investigate the potentials of an AM

\section{WES MCGEE}

University of Michigan approach with nonwoven textile as composite material. In some cases, a nonwoven thermoplastic textile was used together with other natural felt materials to both create varied ribbed textures and to enhance overall stiffness by heat setting after felting.

The prototypes explored four techniques resulting in different surface textures and form: quilting, shiplap, shingle, and thermo-activated composite. For these techniques, different layers of material could be felted together or onto a foam substrate as an integrated process. Additionally, an automatic tape feed facilitated both shiplap and shingle techniques with unique patterns and varying overlap. Through the research, design, and fabrication of acoustic panels and furniture using the techniques developed, the computational design process negotiates the precision and nuances of robotic felting with the specific material behaviors of nonwoven textiles. Geometric limitations are also incorporated, which respond to the tool's movement and the types of material being felted. 

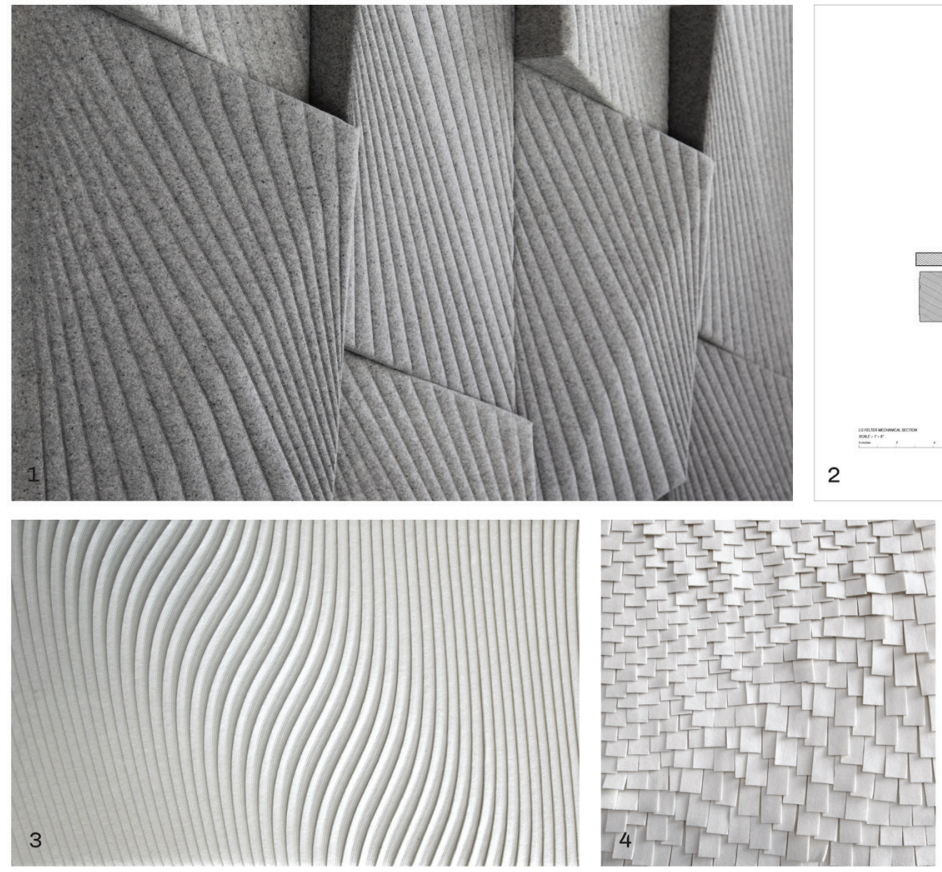
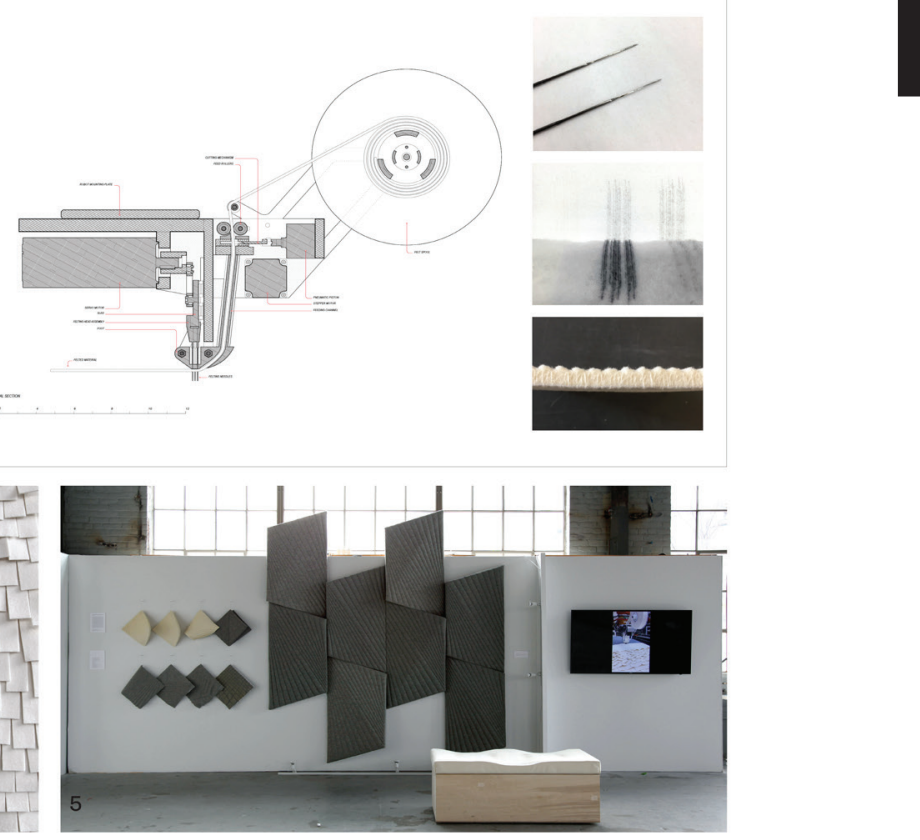

\section{Robotic Needle Felting}

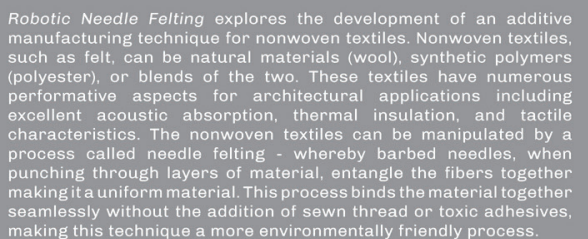

Needle felting can range in scale from handcraft techniques with a single needle to large scale web processing. Integration into a robotic

process not only enables precision and speed in manutacturing
but also extends needle felting as a three-dimensional process especially for surfaces with complex geometries and allowing for homogenoussolid. Througha customized digital workflow, formal and material properties can be varied at local level within a component By developing a fully integrated design to production methodology of potentials for nonwoven textiles in architectural applications.
ons

The project involves three areas of development; the tooling for robotic felting, the digital workflow that enables the formal and
material properties to be specified computationally and embeddec material properties to be specified computationaliy and embedced
into the machine code, and prototypes of architectural elements such as acoustic panels and furniture demonstrating different techniques and processes. Additive manufacturing (AM), commonly known as wide range of disciplines. While AM processes have been developed for a wide range of materials, from ceramics to plastios to metals there have been very few investigations into their applications for
textiles. Given the unique capacity of felt to be seamlessly "addec into a cohesive solid, it presents a unique opportunity to investigate the potentials of an AM approach with nonwoven textile as composite material. In some cases, a nonwoven thermoplastic textile was use textures and to enhance overall stiffness by heat setting after felting

The prototypes explored four techniques resulting in different surface textures and form: quilting, shiplap, shingle, and thermomaterial could be felted together or onto a foam substrate as an integrated process. Additionally, an automatic tape feed facilitat varying overlap. Through the research, design, and fabrication of acoustic panels and furniture using the techniques developed, the computational design process negotiates the precision and nuances textiles. Geometric limitations are also incorporated, which respor to the tools movemont and the typos of material being felted.

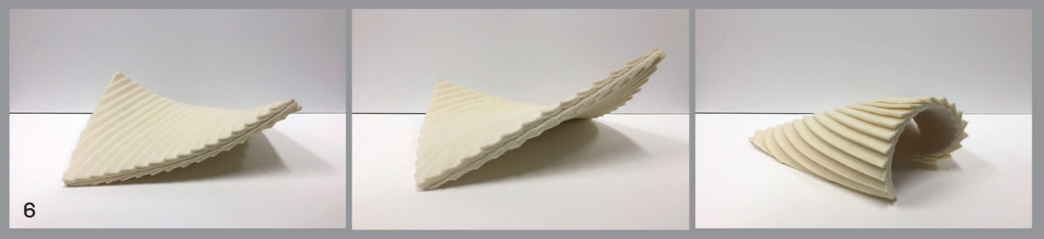

1. Gray quilted texture acoustic panels. $\quad$ 5. View of exhibition (from left to right), samples Credits:

2 Section drawing of end effector for robot, panels, video footage of various robotic felting Design Team: Wes McGee. Tsz Yan Ng. Asa showing automatic pneumatic feed and cut, process, and foreground, bench with felted Peller
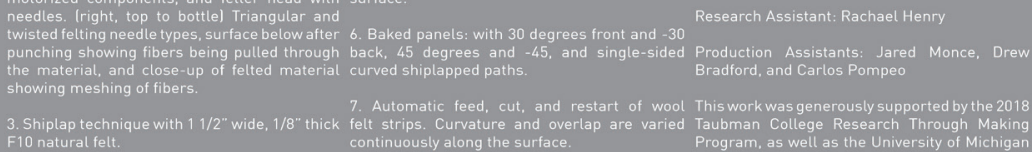

$11 / 2^{\prime \prime \prime}$ wide, $1 / 8$ "thick 8. Approximately $2 \mathrm{ft} \times 2 \mathrm{ft}$ complex surface pou

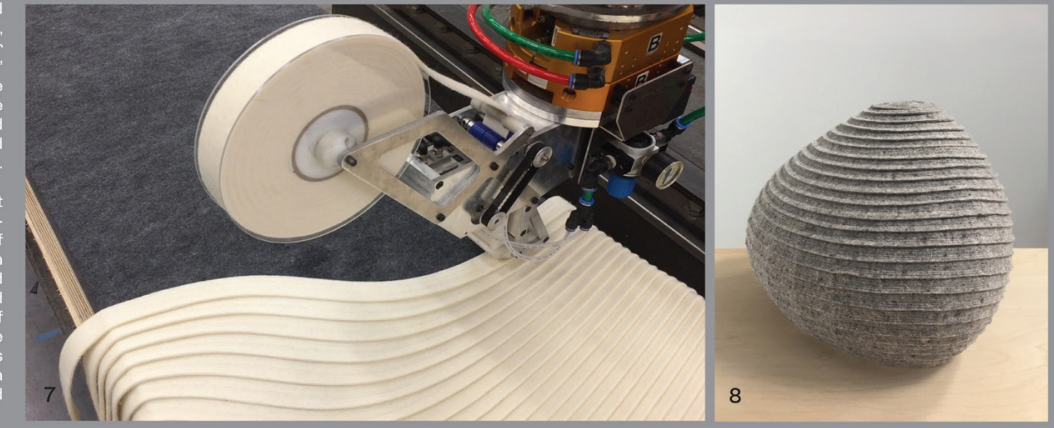

